Research Article

\title{
Experimental and Theoretical Investigations of Terahertz Spectra of the Structural Isomers: Mannose and Galactose
}

\author{
Tongjun Zhang $\mathbb{D}^{1},{ }^{1}$ Shasha Yan $\mathbb{D}^{2},{ }^{2}$ Jianjun Hao $\mathbb{D}^{1},{ }^{1}$ and Dehua Li $\mathbb{D D}^{1}$ \\ ${ }^{1}$ College of Electronic and Information Engineering, Shandong University of Science and Technology, Qingdao 266590, China \\ ${ }^{2}$ College of Energy and Mining Engineering, Shandong University of Science and Technology, Qingdao 266590, China
}

Correspondence should be addressed to Dehua Li; jcbwl@sdust.edu.cn

Received 19 September 2021; Accepted 15 October 2021; Published 26 October 2021

Academic Editor: Vincenza Crupi

Copyright $(02021$ Tongjun Zhang et al. This is an open access article distributed under the Creative Commons Attribution License, which permits unrestricted use, distribution, and reproduction in any medium, provided the original work is properly cited.

The high-resolution terahertz spectra of the two structural isomers, mannose and galactose, have been measured by terahertz time-domain spectroscopy (THz-TDS) in the range of $0.5-4.0 \mathrm{THz}$ at room temperature. Significant differences between these similar molecules have been found in their THz characteristic spectra, implying that THz-TDS is a powerful tool for identifying isomers. Structural analyses and normal mode calculations of the two systems were performed using solid-state density functional theory (DFT) with the PBE and PW91 density functionals as well as using gas-state DFT with B3LYP hybrid functional. Among these calculations, the solid-state simulated results obtained from the PBE method exhibit a good agreement with the experimentally measured spectra. According to the calculated results of PBE, the observed spectral features were assigned as primarily external lattice translations, deformations, and rotations with lesser contributions due to intramolecular motion of pyranose ring, $\mathrm{CH}_{2} \mathrm{OH}$ group, and hydroxyl groups.

\section{Introduction}

Due to the nondestructive and fingerprint properties, terahertz time-domain spectroscopy (THz-TDS) has been established as a promising technique for the study of molecules in the solid state. In recent years, terahertz experimental methods have been widely applied in detecting biological and chemical molecules such as amino acids [1-3], saccharides [4, 5], DNA nucleobases [6], and even harmful and dangerous materials such as pesticides $[7,8]$, narcotics [9], and explosives [10]. The reasonable explanation for these popular applications is that THz-TDS is highly sensitive to similar materials with relatively subtle differences [11]. Meanwhile, an increasing number of researches have been done, and it was found that most organic molecules in solid state have rich and distinct spectra in the frequency region among $0.1-6.0 \mathrm{THz}[12]$.

It is well known that the origins of the $\mathrm{THz}$ spectra are generally attributed to their vibrational modes, which are mostly caused by noncovalent interactions, such as hydrogen bonds and dispersive forces, including crystal lattice vibrations, long-range intramolecular, and intermolecular vibrations as well as combinations of these motions [13]. Thus, the assignment of the experimental $\mathrm{THz}$ spectral features to specific molecular motions is a significant challenge. Recent studies have shown that solid-state density functional theory (DFT) is an excellent means for the complete assignment of the calculated modes to their corresponding experimental $\mathrm{THz}$ spectral features [14]. The inclusion of a crystal packing arrangement in solid-state DFT simulations has led to the accurate reproduction of the external crystal lattice vibrations in addition to the internal molecular motions typically seen in the $\mathrm{THz}$ region [15].

Mannose and galactose are two kinds of monosaccharides that play an important role in human physiological tissues [16]. Mannose is indispensable in human metabolism, especially in the glycosylation of specific proteins. Galactose, which is often found in brain and nerve tissue in the form of galactoside, is also an essential component of some glycoproteins. Mannose and galactose are close structural isomers that have the same molecular formula, $\mathrm{C}_{6} \mathrm{H}_{12} \mathrm{O}_{6}$, but a different arrangement of atoms involved. 
The atom labeling scheme for the mannose molecule is provided in Figure 1(a), as an example. In this study, the experimental $\mathrm{THz}$ absorption spectra from 0.5 to $4.0 \mathrm{THz}$ for mannose and galactose are presented along with a complete computational analysis by using solid- and gas-state DFT. In addition to our previous work [17], another $\mathrm{THz}$ study of these two isomers has been reported by Du et al. [18] with quantitative analysis of isomer mixtures by using PLS and SVR methods. However, previous works focused primarily on either the experimental measurements only or just the theoretical calculations based on the isolated molecules and did not provide calculations for unit cells as well as the mode assignments reported here. In addition to the aforementioned spectral mode descriptions, complete structural analyses of the two isomers were carried out for the experimental structures compared to the calculated data. The study shows that the solid-state DFT calculations can provide good reproduction of the structures and spectra of substances and can accurately explain the subtle differences in the terahertz spectra of the isomers studied here.

\section{Materials and Methods}

2.1. Sample Preparation. Mannose and galactose (purity $\geq 99 \%$ ) were purchased from Shanghai Macklin Co. Ltd. and used without further purification. Both samples were mixed with polytetrafluoroethylene (PTFE) powder at a mass ratio of 1:10 and pulverized using a pestle and mortar to minimize particle size, thereby reducing both Mie scattering and crystal anisotropy. Approximately $350 \mathrm{mg}$ of the sample mixtures were pressed into $13 \mathrm{~mm}$-diameter pellets with a thickness of $1.0 \mathrm{~mm}$ by applying a pressure of $12 \mathrm{MPa}$ for 5 minutes.

2.2. Experimental Apparatus. The experimental apparatus used in this study was a typical THz-TDS setup developed by BATOP Corporation (TDS1008, GER), as illustrated in Figure 2. A mode-locked Ti:sapphire femtosecond laser (MaiTai, Spectra-Physics), with the central wavelength of $780 \mathrm{~nm}$, provides pulses of $100 \mathrm{fs}$ duration with a repetition rate of $80 \mathrm{MHz}$ and an average power of $1.5 \mathrm{~W}$. The emitted laser was separated into a pump beam and a probe beam by a polarized beam splitter (PBS). The pump beam $(11.8 \mathrm{~mW})$ is guided through the fast optical delay line module and then focused to the gap of a low-temperature grown GaAs photoconductive antenna and then generated $\mathrm{THz}$ wave. The terahertz signal is collected and directed to the sample for transmission measurement. The transmitted signal with sample information is then focused onto another photoconductive antenna together with the probe beam $(11.5 \mathrm{~mW})$ for coherent detection. The time delay stage is scanned over a distance of $30 \mathrm{~mm}$ to provide a spectral resolution of $2.0 \mathrm{GHz}$. Dry nitrogen gas was continuously purged into the sample compartment before and during the measurements to minimize the influence of water vapor in the air. The relative humidity is lower than $3 \%$, and the ambient temperature is at $293 \mathrm{~K}$.
2.3. Data Processing. In this study, the optical parameters of the samples were extracted based on the methods developed by Dorney and Duvillaret $[19,20]$. Then the real refractive index $n(\omega)$ and the absorption coefficient $\alpha(\omega)$ can be calculated from the following formula:

$$
\begin{aligned}
& n(\omega)=\frac{\varphi(\omega) c}{\omega d}+1, \\
& \alpha(\omega)=\frac{2 \kappa(\omega) \omega}{c}=\frac{2}{d} \ln \frac{4 n(\omega)}{A(\omega)(n(\omega)+1)^{2}},
\end{aligned}
$$

where $\kappa(\omega)$ is the extinction coefficient, $\omega$ is the angular frequency, $d$ is the sample thickness, and $\mathrm{c}$ is the speed of light in vacuum.

2.4. Theoretical Methods. The total geometry optimization and energy calculations of solid-state mannose and galactose were performed using the Cambridge Sequential Total Energy Package (CASTEP) program [21], which is a part of the Materials Studio package from Accelrys. All calculations were performed based on the fixed unit cells reported by the $\mathrm{X}$-ray diffraction studies obtained from the Cambridge Structural Database. The crystalline unit cell for mannose is provided in Figure 1(b). The unit cell parameters were taken from the published $295 \mathrm{~K}$ crystallographic structures of mannose [22] $(a=5.577 \AA, b=7.5481 \AA, c=18.060 \AA$, $a=\beta=\gamma=90^{\circ}$, and $\left.Z=4\right)$ and galactose [23] $(a=15.7806 \AA$, $b=7.8783 \AA, c=5.9436 \AA, a=\beta=\gamma=90^{\circ}$, and $\left.Z=4\right)$. Both unit cells are orthorhombic with the same space group $\mathrm{P} 22_{1} 2_{1}$. The Perdew-Burke-Ernzerhof (PBE) exchangecorrelation functional [24] and the norm-conserving pseudopotential were utilized in the density functional theory (DFT) calculations within the generalized gradient approximation (GGA). The line search of the BroydenFletcher-Goldfarb-Shanno (BFGS) algorithm was employed in the geometry optimization, and the quality of convergence tolerance was set as "fine." The plane-wave cutoff energy was set as $750 \mathrm{eV}$; Brillouin zone samplings of electronic states were performed on $3 \times 2 \times 1$ and $1 \times 2 \times 2$ Monkhorst-Pack grids [25]. The total energy was converged to $10^{-6} \mathrm{eV} /$ atom, and the atomic coordinates were optimized until the maximum forces between atoms were less than $0.03 \mathrm{eV} / \AA$. The grids for fast Fourier transform of mannose and galactose were $45 \times 60 \times 144$ and $125 \times 64 \times 48$, respectively. Perdew-Wang91 (PW91) [26] calculations were also implemented on both unit cells in the same settings.

For comparison, calculations on isolated molecules of the isomers were carried out using the gaseous state theory within the DFT method. The B3LYP functional together with the 6-311+G (d, p) Gaussian-type basis set was performed with the program option "tight" convergence criteria.

\section{Results and Discussion}

3.1. Structural Analysis. The DFT calculated values and $\mathrm{X}$-ray experimental structural data for mannose and galactose are presented in Table 1(length), 2 (angles), and 3 (HB length). The structural data were obtained from the 


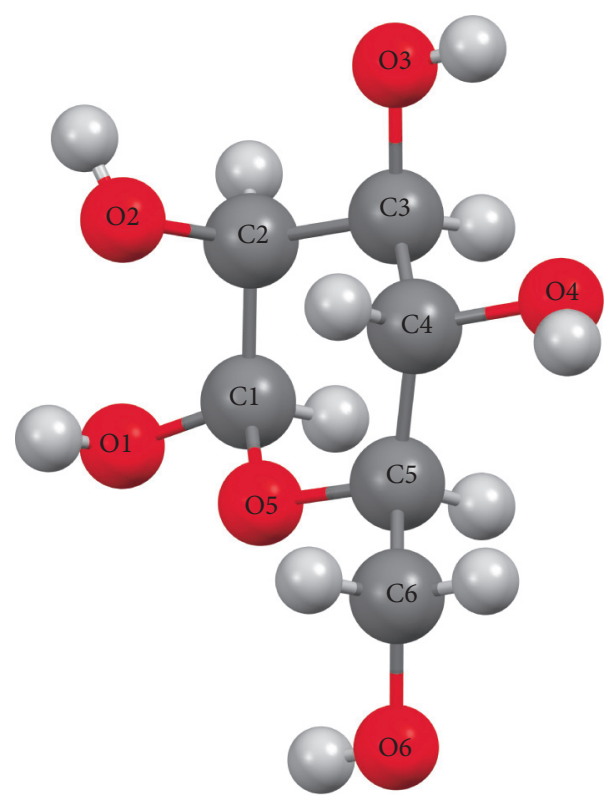

(a)

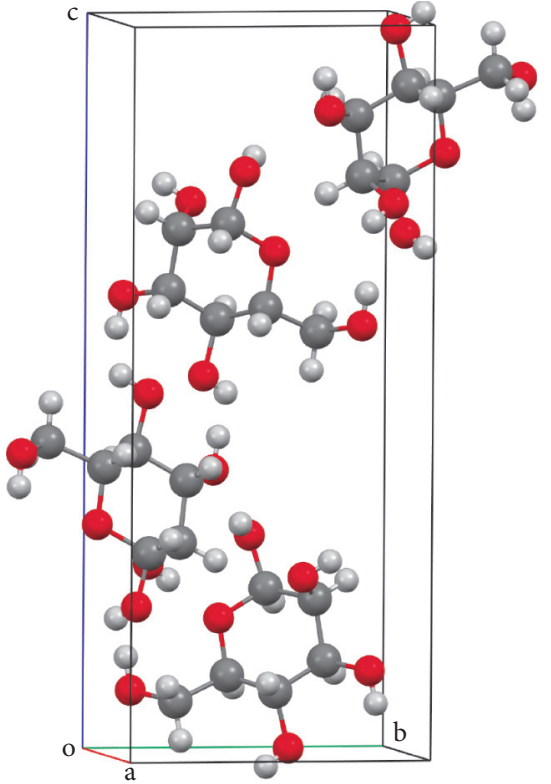

(b)

Figure 1: (a) Atom labeling scheme for mannose in the form of zwitterion and (b) molecular packing in the unit cell. Atom colors: oxygen, red; carbon, grey; and hydrogen, white. Only the mannose molecule is depicted in the figure, for the reason that the labeling schemes for mannose and galactose are the same as these molecules are structurally equivalent, differing only in the spatial position of the four chiral carbons.

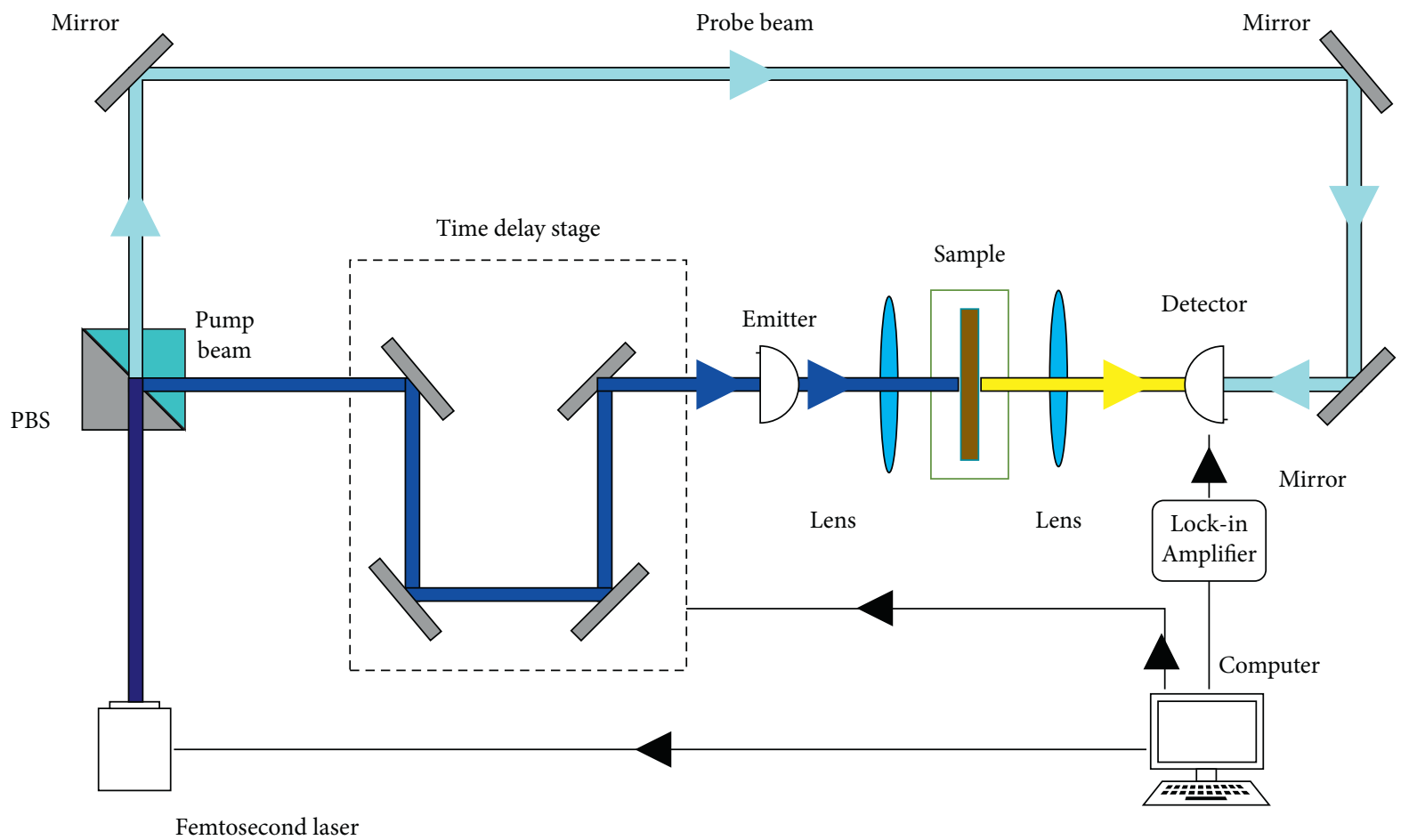

FIgURE 2: The schematic diagram of the THz-TDS system.

geometry optimized structures and compared with the experimental crystallographic results to evaluate the quality of the various calculations in terms of root-mean-squared deviations (RMSDs).
As shown in Table 1, the bond lengths provided by the B3LYP functional with $6-311+\mathrm{G}(\mathrm{d}, \mathrm{p})$ basis set are in best agreement with the experiment, yielding RMSD values of 0.0258 and 0.0142 for mannose and galactose, respectively. 
TABLE 1: Calculated bond lengths $(\AA)$ and RMSD values of mannose and galactose compared to experimental X-ray values ${ }^{\mathrm{a}}$.

\begin{tabular}{|c|c|c|c|c|c|c|c|c|c|}
\hline \multicolumn{5}{|c|}{ Mannose } & \multicolumn{5}{|c|}{ Galactose } \\
\hline \multicolumn{2}{|c|}{ CASTEP } & \multicolumn{5}{|c|}{ Gaussian } & \multicolumn{2}{|c|}{ CASTEP } & \multirow{2}{*}{$\begin{array}{c}\text { Gaussian } \\
\text { B3LYP }\end{array}$} \\
\hline Bond & Exp. & PBE & PW91 & B3LYP & Bond & Exp. & PBE & PW91 & \\
\hline $\mathrm{C}_{1}-\mathrm{O}_{1}$ & 1.3687 & 1.3891 & 1.3912 & 1.3816 & $\mathrm{C}_{2}-\mathrm{O}_{1}$ & 1.4002 & 1.4261 & 1.4259 & 1.4095 \\
\hline $\mathrm{C}_{1}-\mathrm{O}_{5}$ & 1.4546 & 1.4467 & 1.4447 & 1.4326 & $\mathrm{C}_{2}-\mathrm{O}_{5}$ & 1.4326 & 1.4120 & 1.4114 & 1.4084 \\
\hline $\mathrm{C}_{1}-\mathrm{C}_{2}$ & 1.4959 & 1.5368 & 1.5399 & 1.5380 & $\mathrm{C}_{3}-\mathrm{O}_{2}$ & 1.4420 & 1.4299 & 1.4304 & 1.4392 \\
\hline $\mathrm{C}_{2}-\mathrm{O}_{2}$ & 1.3899 & 1.4242 & 1.4246 & 1.4230 & $\mathrm{C}_{4}-\mathrm{O}_{3}$ & 1.4254 & 1.4313 & 1.4318 & 1.4141 \\
\hline $\mathrm{C}_{2}-\mathrm{C}_{3}$ & 1.5365 & 1.5380 & 1.5411 & 1.5266 & $\mathrm{C}_{1}-\mathrm{O}_{4}$ & 1.4307 & 1.4319 & 1.4329 & 1.4222 \\
\hline $\mathrm{C}_{3}-\mathrm{O}_{3}$ & 1.4263 & 1.4288 & 1.4322 & 1.4238 & $\mathrm{C}_{5}-\mathrm{O}_{5}$ & 1.4260 & 1.4466 & 1.4465 & 1.4455 \\
\hline $\mathrm{C}_{3}-\mathrm{C}_{4}$ & 1.4793 & 1.5266 & 1.5292 & 1.5238 & $\mathrm{C}_{6}-\mathrm{O}_{6}$ & 1.4193 & 1.4322 & 1.4322 & 1.4223 \\
\hline $\mathrm{C}_{4}-\mathrm{O}_{4}$ & 1.4324 & 1.4280 & 1.4291 & 1.4322 & $\mathrm{C}_{2}-\mathrm{C}_{3}$ & 1.5361 & 1.5362 & 1.5352 & 1.5466 \\
\hline $\mathrm{C}_{4}-\mathrm{C}_{5}$ & 1.4894 & 1.5434 & 1.5462 & 1.5326 & $\mathrm{C}_{3}-\mathrm{C}_{4}$ & 1.5016 & 1.5311 & 1.5404 & 1.5284 \\
\hline $\mathrm{C}_{5}-\mathrm{O}_{5}$ & 1.4197 & 1.4365 & 1.4410 & 1.4292 & $\mathrm{C}_{1}-\mathrm{C}_{4}$ & 1.5442 & 1.5431 & 1.5419 & 1.5344 \\
\hline $\mathrm{C}_{5}-\mathrm{C}_{6}$ & 1.5181 & 1.5209 & 1.5211 & 1.5230 & $\mathrm{C}_{1}-\mathrm{C}_{5}$ & 1.5378 & 1.5410 & 1.5407 & 1.5329 \\
\hline $\mathrm{C}_{6}-\mathrm{O}_{6}$ & 1.4338 & 1.4245 & 1.4305 & 1.4135 & $\mathrm{C}_{5}-\mathrm{C}_{6}$ & 1.5117 & 1.5231 & 1.5315 & 1.5261 \\
\hline RMSD & - & 0.0272 & 0.0289 & 0.0258 & & - & 0.0155 & 0.0178 & 0.0142 \\
\hline
\end{tabular}

${ }^{a}$ The experimental values are taken from references $[22,23]$.

Among the 12 bond lengths listed in Table 1, the values of mannose simulated by PBE and PW91 each have nine bonds that are slightly larger than their corresponding experimental values. In contrast, the values of galactose calculated by PBE and PW91 have nine and eight bonds, respectively, which are slightly larger than their corresponding experimental values. Therefore, a little overestimation of bond lengths was appeared in the solid-state simulations, producing RMSD values of 0.0272 and 0.0289 for mannose, and 0.0155 and 0.0178 for galactose for the PBE and PW91 calculations, respectively. The most significant bias in calculated bond lengths happened on overestimating the $\mathrm{C}_{4}-\mathrm{C}_{5}$ bond in mannose with the deviation by $0.0568 \AA$ and the $\mathrm{C}_{3}-\mathrm{C}_{4}$ bond in galactose with the deviation by $0.0388 \AA$ using the PW91 calculations. The $\mathrm{C}_{1}-\mathrm{O}_{5}$ bond in mannose and $\mathrm{C}_{2}-\mathrm{O}_{5}$ bond in galactose were clearly underestimated in the calculations using B3LYP functional with the deviation of $0.0220 \AA$ and $0.0242 \AA$, respectively. The minimum bias occurred in the $\mathrm{C}_{2}-\mathrm{C}_{3}$ bond in galactose from the PBE calculation with the deviation by $0.0001 \AA$, while with the deviation by $0.0105 \AA$ from the B3LYP calculation. The simulations of the PW91 functional generally tend to overestimate the bond length, while the B3LYP functional generally underestimate the bond length.

As shown in Table 2, the PBE density functional provided the best reproduction of the bond angles in the three calculations, yielding the smallest RMSD values of 1.168 and 2.050 for mannose and galactose, respectively. In the B3LYP simulation, there is an obvious tendency to underestimate the bond angle. The maximum RMSD values of mannose and galactose are 2.219 and 2.285, respectively. The most significant deviation in the calculated bond angles occurred in the underestimation of the $\mathrm{O}_{2}-\mathrm{C}_{3}-\mathrm{C}_{4}$ bond angle in galactose using B3LYP calculation employing 6-311+G (d, p) basis set. Furthermore, $\mathrm{C}_{1}-\mathrm{C}_{2}-\mathrm{C}_{3}$ bond angle in mannose was mainly overestimated in the calculation using B3LYP functional. Although the best reproduction of bond lengths in both saccharides was at the B3LYP/6-311+G $(\mathrm{d}, \mathrm{p})$ level, as indicated by the comparatively low RMSD of 0.0258 and 0.0142. Because the most considerable bias between the calculated and experimental bond angles occurred in both monosaccharides, the favorite predictions cannot be achieved by using gas-state simulation at B3LYP functional level.

Hydrogen bonds formed by the saccharide molecules in unit cells are the primary intermolecular interactions that have a great influence on the observed features of $\mathrm{THz}$ spectra. Therefore, the high-quality reproduction of hydrogen bond length in DFT calculations is essential for the effective simulation of $\mathrm{THz}$ spectra. The hydrogen bond lengths obtained from DFT calculations and experiments are provided in Table 3. Only one type of hydrogen bond $\mathrm{O} \cdots \mathrm{H}-\mathrm{O}$ exists in hydrogen-bonding systems, which involve the five hydroxyl groups of each molecule of both solids. The oxygen atoms act as a donor and an acceptor except for the pyranose ring $\mathrm{O}$. The hydrogen bond lengths calculated at the PBE functional level were in better agreement with the experiment values for both mannose and galactose, with RMSD values of 0.0426 and $0.1284 \AA$, respectively. The highest RMSD value of both systems is $0.063 \AA$, which was obtained from the PW91 calculation. Only subtle RMSD variation was observed between the PBE and PW91 functionals, producing values of 0.005 and 0.007 for mannose and galactose, respectively. Except for $\mathrm{O}_{6} \cdots \mathrm{H}_{4}-\mathrm{O}_{1}$, almost all the calculated hydrogen bond lengths in galactose simulations were underestimated. The biggest deviations appeared in the underestimation of the $\mathrm{O}_{1} \cdots \mathrm{H}_{11}-\mathrm{O}_{3}$ hydrogen bond length with a difference as high as $0.237 \AA$ in the PW91 calculation for galactose. Under the comprehensive consideration of the whole structural data and the comparison of RMSD values, the overall molecular structure of mannose and galactose are most accurately reproduced by solid-state calculations using PBE functional.

\subsection{The Terahertz Spectra and Vibrational Modes Assignment.}

The terahertz absorption spectra of mannose and galactose measured by $\mathrm{THz}$-TDS at room temperature $293 \mathrm{~K}$ are shown in Figure 3. Both spectra have well- 
TABLE 2: Calculated bond angles $\left({ }^{\circ}\right)$ and RMSD values of mannose and galactose compared to experimental X-ray values ${ }^{\mathrm{a}}$.

\begin{tabular}{|c|c|c|c|c|c|c|c|c|c|}
\hline \multicolumn{5}{|c|}{ Mannose } & \multicolumn{5}{|c|}{ Galactose } \\
\hline \multicolumn{2}{|c|}{ CASTEP } & \multirow{2}{*}{$\begin{array}{c}\text { Gaussian } \\
\text { PBE }\end{array}$} & \multicolumn{2}{|c|}{ CASTEP } & \multirow{2}{*}{$\begin{array}{c}\text { Gaussian } \\
\text { Bond angle }\end{array}$} & \multirow[b]{2}{*}{ Exp. } & \multirow[b]{2}{*}{ PBE } & \multirow[b]{2}{*}{ PW91 } & \multirow[b]{2}{*}{ B3LYP } \\
\hline Bond angle & Exp. & & PW91 & B3LYP & & & & & \\
\hline $\mathrm{O}_{1}-\mathrm{C}_{1}-\mathrm{O}_{5}$ & 105.60 & 106.28 & 106.82 & 108.25 & $\mathrm{O}_{1}-\mathrm{C}_{2}-\mathrm{O}_{5}$ & 111.83 & 110.61 & 110.61 & 109.84 \\
\hline $\mathrm{O}_{1}-\mathrm{C}_{1}-\mathrm{C}_{2}$ & 115.70 & 113.76 & 113.94 & 111.16 & $\mathrm{O}_{1}-\mathrm{C}_{2}-\mathrm{C}_{3}$ & 108.11 & 112.12 & 112.47 & 110.17 \\
\hline $\mathrm{O}_{5}-\mathrm{C}_{1}-\mathrm{C}_{2}$ & 111.00 & 109.66 & 110.06 & 110.72 & $\mathrm{C}_{3}-\mathrm{C}_{2}-\mathrm{O}_{5}$ & 107.55 & 110.74 & 110.82 & 111.24 \\
\hline $\mathrm{O}_{2}-\mathrm{C}_{2}-\mathrm{C}_{1}$ & 106.30 & 108.50 & 108.59 & 106.63 & $\mathrm{C}_{2}-\mathrm{C}_{3}-\mathrm{C}_{4}$ & 110.07 & 108.72 & 108.80 & 111.67 \\
\hline $\mathrm{O}_{2}-\mathrm{C}_{2}-\mathrm{C}_{3}$ & 112.20 & 112.50 & 112.54 & 110.62 & $\mathrm{C}_{2}-\mathrm{C}_{3}-\mathrm{O}_{2}$ & 109.34 & 108.89 & 108.75 & 109.97 \\
\hline $\mathrm{C}_{1}-\mathrm{C}_{2}-\mathrm{C}_{3}$ & 107.60 & 108.53 & 109.06 & 111.88 & $\mathrm{O}_{2}-\mathrm{C}_{3}-\mathrm{C}_{4}$ & 112.90 & 113.79 & 113.84 & 106.72 \\
\hline $\mathrm{O}_{3}-\mathrm{C}_{3}-\mathrm{C}_{4}$ & 111.80 & 111.28 & 111.87 & 111.45 & $\mathrm{C}_{3}-\mathrm{C}_{4}-\mathrm{C}_{1}$ & 109.77 & 108.88 & 108.78 & 109.77 \\
\hline $\mathrm{O}_{3}-\mathrm{C}_{3}-\mathrm{C}_{2}$ & 107.60 & 109.58 & 108.78 & 107.04 & $\mathrm{C}_{3}-\mathrm{C}_{4}-\mathrm{O}_{3}$ & 110.79 & 111.71 & 111.69 & 111.15 \\
\hline $\mathrm{C}_{4}-\mathrm{C}_{3}-\mathrm{C}_{2}$ & 112.90 & 112.12 & 112.36 & 111.13 & $\mathrm{O}_{3}-\mathrm{C}_{4}-\mathrm{C}_{1}$ & 110.14 & 112.74 & 112.78 & 110.35 \\
\hline $\mathrm{O}_{4}-\mathrm{C}_{4}-\mathrm{C}_{3}$ & 106.20 & 107.02 & 106.82 & 105.92 & $\mathrm{C}_{4}-\mathrm{C}_{1}-\mathrm{C}_{5}$ & 107.75 & 107.48 & 107.49 & 107.18 \\
\hline $\mathrm{O}_{4}-\mathrm{C}_{4}-\mathrm{C}_{5}$ & 111.80 & 111.00 & 111.27 & 113.37 & $\mathrm{C}_{4}-\mathrm{C}_{1}-\mathrm{O}_{4}$ & 110.79 & 112.90 & 112.79 & 112.58 \\
\hline $\mathrm{C}_{3}-\mathrm{C}_{4}-\mathrm{C}_{5}$ & 110.90 & 110.08 & 109.34 & 109.02 & $\mathrm{O}_{4}-\mathrm{C}_{1}-\mathrm{C}_{5}$ & 113.36 & 113.26 & 113.24 & 110.73 \\
\hline $\mathrm{O}_{5}-\mathrm{C}_{5}-\mathrm{C}_{4}$ & 111.70 & 111.46 & 110.86 & 108.63 & $\mathrm{C}_{1}-\mathrm{C}_{5}-\mathrm{O}_{5}$ & 110.64 & 111.04 & 111.14 & 107.79 \\
\hline $\mathrm{O}_{5}-\mathrm{C}_{5}-\mathrm{C}_{6}$ & 107.00 & 105.89 & 106.71 & 105.56 & $\mathrm{C}_{1}-\mathrm{C}_{5}-\mathrm{C}_{6}$ & 113.15 & 115.73 & 115.59 & 114.15 \\
\hline $\mathrm{C}_{4}-\mathrm{C}_{5}-\mathrm{C}_{6}$ & 111.50 & 112.61 & 113.04 & 114.44 & $\mathrm{O}_{5}-\mathrm{C}_{5}-\mathrm{C}_{6}$ & 105.42 & 103.31 & 103.29 & 107.00 \\
\hline $\mathrm{O}_{6}-\mathrm{C}_{6}-\mathrm{C}_{5}$ & 110.80 & 111.54 & 112.35 & 111.30 & $\mathrm{C}_{5}-\mathrm{C}_{6}-\mathrm{O}_{6}$ & 109.18 & 113.13 & 113.19 & 111.02 \\
\hline $\mathrm{C}_{5}-\mathrm{O}_{5}-\mathrm{C}_{1}$ & 112.20 & 113.43 & 112.76 & 114.44 & $\mathrm{C}_{2}-\mathrm{O}_{5}-\mathrm{C}_{5}$ & 114.22 & 115.24 & 115.41 & 113.87 \\
\hline RMSD & - & 1.168 & 1.179 & 2.219 & RMSD & - & 2.050 & 2.102 & 2.285 \\
\hline
\end{tabular}

${ }^{a}$ The experimental values are taken from references $[22,23]$.

TABLE 3: Calculated hydrogen-bond lengths $(\AA)$ and RMSD values of mannose and galactose compared to experimental X-ray values ${ }^{\mathrm{a}}$.

\begin{tabular}{|c|c|c|c|c|c|c|c|c|c|}
\hline \multirow[b]{2}{*}{ NO. } & \multicolumn{2}{|c|}{ Mannose } & \multicolumn{6}{|c|}{ Galactose } & \multirow[b]{2}{*}{ PW91 } \\
\hline & H-bond & Exp. & PBE & PW91 & NO. & H-bond & Exp. & PBE & \\
\hline 1 & $\mathrm{O}_{1} \cdots \mathrm{H}_{12}-\mathrm{O}_{6}$ & 2.757 & 2.749 & 2.750 & 1 & $\mathrm{O}_{1} \cdots \mathrm{H}_{11}-\mathrm{O}_{3}$ & 3.111 & 2.883 & 2.874 \\
\hline 2 & $\mathrm{O}_{1} \cdots \mathrm{H}_{8}-\mathrm{O}_{3}$ & 2.843 & 2.848 & 2.805 & 2 & $\mathrm{O}_{2} \cdots \mathrm{H}_{12}-\mathrm{O}_{6}$ & 2.748 & 2.727 & 2.716 \\
\hline 3 & $\mathrm{O}_{2} \cdots \mathrm{H}_{9}-\mathrm{O}_{5}$ & 2.861 & 2.849 & 2.882 & 3 & $\mathrm{O}_{3} \cdots \mathrm{H}_{2}-\mathrm{O}_{4}$ & 2.915 & 2.775 & 2.76 \\
\hline 4 & $\mathrm{O}_{3} \cdots \mathrm{H}_{10}-\mathrm{O}_{4}$ & 3.092 & 2.998 & 3.188 & 4 & $\mathrm{O}_{4} \cdots \mathrm{H}_{3}-\mathrm{O}_{2}$ & 2.888 & 2.857 & 2.846 \\
\hline 5 & $\mathrm{O}_{6} \cdots \mathrm{H}_{11}-\mathrm{O}_{4}$ & 2.693 & 2.698 & 2.696 & 5 & $\mathrm{O}_{6} \cdots \mathrm{H}_{4}-\mathrm{O}_{1}$ & 2.662 & 2.759 & 2.757 \\
\hline & RMSD & - & 0.0426 & 0.0472 & & RMSD & - & 0.1284 & 0.1357 \\
\hline
\end{tabular}

${ }^{\mathrm{a}}$ The experimental values are taken from references $[22,23]$.

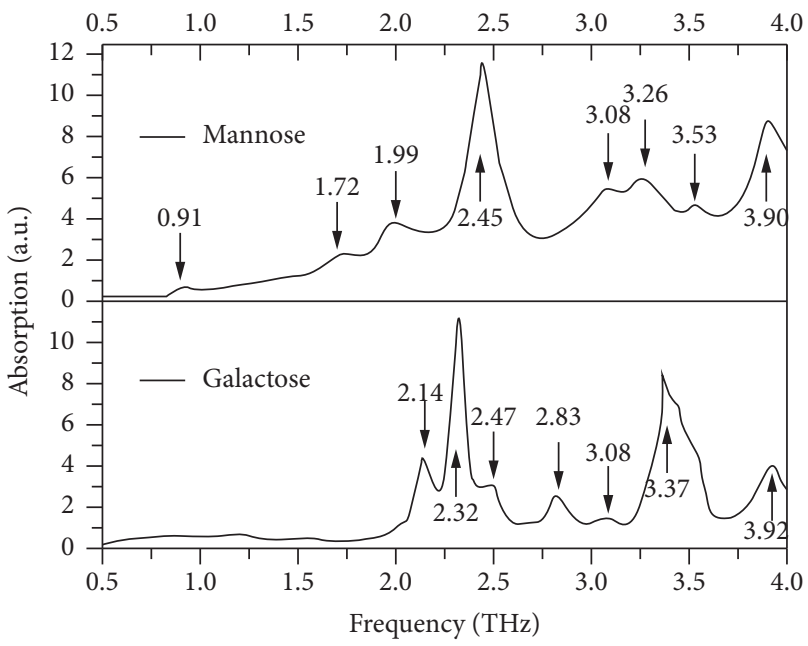

FIGURE 3: The comparison of the experimental terahertz absorption spectra of mannose and galactose.

resolved absorption peaks in the frequency range of 0.5 to $4.0 \mathrm{THz}$. For mannose, eight absorption features were obtained at the following frequencies: $0.91,1.72,1.99$,
$2.45,3.08,3.26,3.53$, and $3.90 \mathrm{THz}$. Among these features, the strongest absorption locates at $2.45 \mathrm{THz}$. While the features that occurred at $0.91,1.72$, and $3.53 \mathrm{THz}$ can be recognized as weak-intensity features; the other four features are considered as medium-intensity types. For galactose, seven absorption peaks were observed in the experiment, which were all above $2.0 \mathrm{THz}$. Two sharp and strong features are located at 2.32 and $3.37 \mathrm{THz}$; a weak feature occurs at $3.08 \mathrm{THz}$. The remaining three features located at 2.14, 2.47, 2.83, and $3.92 \mathrm{THz}$ can be considered as medium-intensity features. For the reason that the isomers are very similar in molecular formula and spatial structure, which determines that their terahertz absorption spectra share certain similar characteristics. For example, the absorption peak in intensity at $3.90 \mathrm{THz}$ of mannose corresponds well with $3.92 \mathrm{THz}$ of galactose, where the frequency difference is only $0.02 \mathrm{THz}$ within the spectrum resolution. However, it can be clearly observed that the terahertz spectra of the two isomers are clearly different, which proves that the observed $\mathrm{THz}$ spectrum can be served as a conformational fingerprint, and even very small changes in molecular configuration can lead to remarkable spectral differences. Therefore, it 
can be confirmed that THz-TDS has great potential as an effective means for isomer identification.

In Figure 4, the experimental spectra are compared with the simulated $\mathrm{THz}$ spectra produced by gas- and solid-state DFT calculations for mannose and galactose to evaluate the spectral reproduction capabilities for each of the three functionals. The simulated spectra are shown in stick form with an empirical $3 \mathrm{~cm}^{-1}$ full-width at half-maximum (FWHM) Lorentzian line shape obtained by using the free software Multiwfn3.6 [27]. Gas-state spectra calculated by the B3LYP functional generate three normal modes, which have no good agreement with the experimental features. As can be seen from Figure 4, the simulated spectrum of galactose has a rich absorption peak distribution in the $1.5-4.0 \mathrm{THz}$ region, which is consistent with the spectrum observed in the experiment. All simulations slightly underestimated seven features in the galactose spectrum except for the PBE calculation, which showed the best prediction of the peak locations and relative intensities for the experimental observations. The PBE calculations predict eight IR-active modes in the terahertz region, while there are seven observed features in the experimental $\mathrm{THz}$ spectrum, as shown in Table 4.

The final vibrational modes of mannose and galactose were assigned according to $\mathrm{PBE}$ calculations, which are qualitatively determined by verifying normal mode displacement eigenvectors. Table 4 shows the characteristic absorption peaks obtained from the experimental measurements and PBE calculations, as well as the tentative assignments of the vibrational modes of the two solids. No imaginary frequencies were found in the final calculation results, and no scaling factors were applied to the prediction of vibrational spectra. The predicted vibrational modes in the terahertz range consist mainly of the internal and external motions of the molecules. Generally, the internal modes mainly include the wagging and torsion of pyranose ring, hydroxyl, and $\mathrm{CH}_{2} \mathrm{OH}$ groups, whereas the external modes primarily involve the whole molecular translation, rotation, deformation, and so on.

Eight observed features in the mannose's experimental spectrum were assigned as modes $a$ to $h$ as shown in Table 4. These modes are all primarily of external characteristic motion, with mode $a$ exhibiting $80 \%$ translational and $20 \%$ rotational characters in the overall motion, mode $b$ showing $50 \%$ rotational motion and 50\% translational character, mode $c$ indicating $70 \%$ deformational motion and $30 \%$ internal motion, and so forth. The internal motion of mode $c$ originates from the internal wagging and torsion of the $\mathrm{CH}_{2} \mathrm{OH}$ and hydroxyl groups. The remaining four modes $e$ to $h$ are listed in Table 4 , and their displacement vectors are shown in Figure 5 and discussed in detail thereafter.

Seven features observed in the galactose terahertz spectrum are assigned as modes i to vii, as shown in Table 4. The displacement vector representations of modes i, iii, and iv are analyzed in detail in Figure 6. Mode ii is primarily an external vibration, showing $60 \%$ external translation and $40 \%$ internal contribution from the wagging around the $\mathrm{C}_{2}$ and $\mathrm{C}_{3}$ hydroxyl groups. Mode $\mathrm{v}$ mainly originates from the internal motions caused by the pyranose ring deformation and the $\mathrm{CH}_{2} \mathrm{OH}$ group wagging and the $40 \%$ external contribution of the rotation along the $a$-axis. Mode vi is completely derived from external vibrations along different axes, of which $60 \%$ are translational vibrations along the $a$ axis and $40 \%$ are rotational vibrations along the $b$-axis. Mode vii mostly comes from the external rotation along the $a$-axis and the $30 \%$ internal contribution caused by the pyranose ring torsion and the $\mathrm{CH}_{2} \mathrm{OH}$ group wagging. Modes i, iii, iv, and vi are absent in the B3LYP simulation, indicating that these vibrational modes are purely derived from external vibrations.

Among the comparisons of the three DFT calculations mentioned above, the terahertz spectrum simulated by PBE functional had the best agreement with the observed spectra in terms of both the feature positions and the matching of the infrared intensities. Therefore, the four selected experimental features at 2.93, 3.26, 3.68, and 3.80 THz are assigned according to the calculation results of $\mathrm{PBE}$, although there are still subtle differences between the experimental and the theoretical frequencies. The displacement vector representations for the four chosen assigned modes are shown in Figure 5. The observed absorption features in the terahertz region mainly arise from the collective motions of molecules due to hydrogen bonds, along with some intramolecular motions. The first intense vibrational mode at $3.80 \mathrm{THz}$ is dominated by the external deformations between adjacent molecules. The mode for $2.93 \mathrm{THz}$ is mainly about external deformation together with the external rotations along different axes. The third mode at $3.26 \mathrm{THz}$ partly involves the intramolecular pyranose ring and $\mathrm{CH}_{2} \mathrm{OH}$ group deformation and partly originates from external deformations.

The interaction of galactose molecules in the unit cell and their displacement vector representations of several typical vibrational modes calculated by PBE functional were demonstrated in Figure 6. The galactose molecule is linked to its adjacent molecules and forms a three-dimensional structure through $\mathrm{O} \cdots \mathrm{H}-\mathrm{O}$ hydrogen bonds and $\mathrm{O} \cdots \mathrm{O}$ bonds. Therefore, the crystal structure is primarily stabilized by $\mathrm{O} \cdots \mathrm{H}-\mathrm{O}$ hydrogen bonds and $\mathrm{O} \cdots \mathrm{O}$ bonds. The calculation results show that many characteristic spectra of galactose in the terahertz frequency range belong to lowfrequency collective vibration modes. Still, there are noticeable differences in some specific details. For example, the vibrational modes of galactose at 2.06 and $2.43 \mathrm{THz}$ are all collective. However, the former is more manifested in the external deformation along $a$-axis, while the latter is more reflected in the external rotation of neighboring molecules along $b$-axis. The vibration of galactose at $2.65 \mathrm{THz}$ is part of the external rotation and deformation along different axes. The vibrational mode at $2.79 \mathrm{THz}$ arises mainly from the external translation along the axis.

However, certain differences exist in the interactions between the internal hydrogen bonds of the isomer crystals. As for mannose, the molecule is linked to neighboring molecules through a network of 14 hydrogen bonds [22], while the hydrogen bond network of galactose contains only nine hydrogen bonds [28]. Theoretical analysis reveals that the resonance absorption peaks of monosaccharides in the terahertz band are mainly derived from the diverse collective 


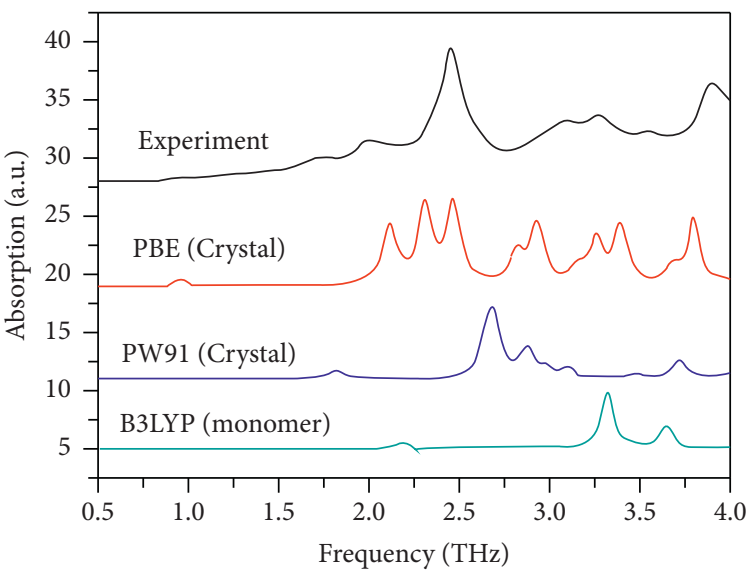

(a)

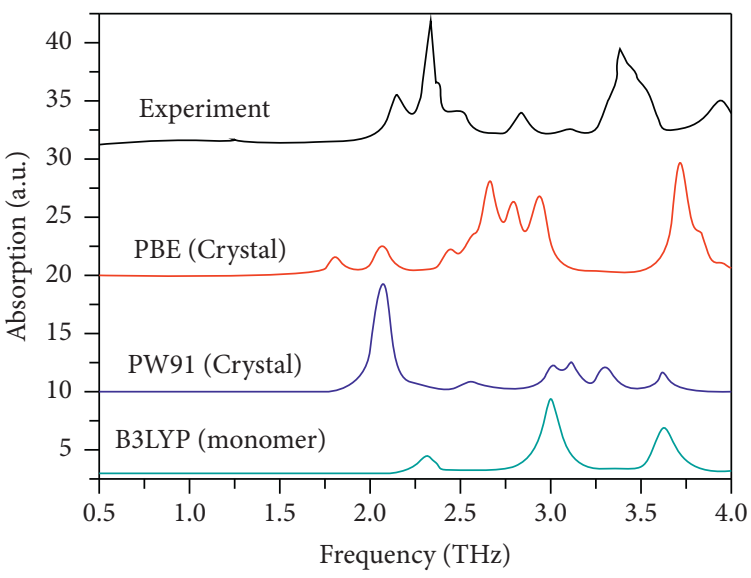

(b)

FIGURE 4: The experimental absorption spectra of (a) mannose and (b) galactose compared with the simulated THz spectra of the solid-state model using PBE and PW91 functionals and that of isolated molecule model using B3LYP functional.

TABLE 4: Comparison of the absorption peaks between the experimental and best-fit calculated spectra and the mode descriptions of mannose and galactose.

\begin{tabular}{|c|c|c|c|}
\hline \multicolumn{4}{|r|}{ Mannose } \\
\hline Mode & Exp. & Cal. & Mode description $^{\mathrm{a}}$ \\
\hline a & 0.91 & 0.94 & $80 \%$ ET along $b$-axis, $20 \%$ ER along $c$-axis \\
\hline $\mathrm{b}$ & 1.72 & & $50 \%$ ET along $a$-axis, $50 \%$ ER along $c$-axis \\
\hline \multirow{2}{*}{ c } & \multirow{2}{*}{1.99} & 2.11 & $60 \%$ ER along $a$-axis, $40 \%$ IM ( $\mathrm{CH}_{2} \mathrm{OH}$ group wagging, hydroxyl groups torsions) \\
\hline & & 2.31 & $85 \%$ ET along $c$-axis, $15 \%$ ER along $a$-axis \\
\hline \multirow{2}{*}{$\mathrm{d}$} & \multirow{2}{*}{2.45} & 2.46 & $75 \%$ ED along $c$-axis, $25 \%$ ED along $a$-axis \\
\hline & & 2.82 & $50 \%$ ET along $c$-axis, $25 \%$ ER along $a$-axis, $25 \%$ ER along $b$-axis. \\
\hline e & 3.08 & 2.93 & $60 \% \mathrm{ED}$ along $c$-axis, $40 \%$ ET along $a$-axis \\
\hline \multirow{2}{*}{$\mathrm{f}$} & \multirow{2}{*}{3.26} & 3.26 & $50 \% \mathrm{ED}$ along $c$-axis, $50 \%$ IM (pyranose ring deformation, $\mathrm{CH}_{2} \mathrm{OH}$ group deformation.) \\
\hline & & 3.39 & $55 \%$ ET along $c$-axis, $45 \%$ ER along $a$-axis \\
\hline g & 3.53 & 3.68 & $50 \%$ ED along $a$-axis, $50 \%$ IM $\left(\mathrm{C}_{4} \mathrm{HOH}\right.$ group wagging, $\mathrm{CH}_{2} \mathrm{OH}$ deformation $)$ \\
\hline $\mathrm{h}$ & 3.90 & 3.80 & $70 \%$ ED along $b$-axis, $30 \%$ ER along $c$-axis \\
\hline \multicolumn{4}{|c|}{ Galactose } \\
\hline \multirow[t]{2}{*}{ Mode } & \multirow[t]{2}{*}{ Exp. } & Cal. & Mode description $^{\mathrm{a}}$ \\
\hline & & 1.80 & $65 \%$ ER along $a$-axis, $35 \%$ ER along $b$-axis \\
\hline $\mathrm{i}$ & 2.14 & 2.06 & $55 \%$ ED along $a$-axis, $45 \%$ ER along $c$-axis \\
\hline ii & 2.32 & & $60 \%$ ET along $a$-axis, $40 \%$ IM ( $\mathrm{C}_{2}$ hydroxyl, $\mathrm{C}_{3}$ hydroxyl groups wagging) \\
\hline \multirow{2}{*}{ iii } & \multirow{2}{*}{2.47} & 2.43 & $75 \%$ ER along $a$-axis, $25 \%$ ET along $c$-axis \\
\hline & & 2.65 & $55 \%$ ER along $b$-axis, $45 \%$ ER along $c$-axis \\
\hline iv & 2.83 & 2.79 & $80 \%$ ET along $a$-axis, $20 \%$ ER along $b$-axis \\
\hline $\mathrm{v}$ & 3.08 & 2.94 & $40 \%$ ER along $a$-axis, $60 \%$ IM (pyranose ring deformation, $\mathrm{CH}_{2} \mathrm{OH}$ group wagging) \\
\hline \multirow{2}{*}{ vi } & \multirow{2}{*}{3.37} & & $60 \%$ ET along $a$-axis, $40 \%$ ER along $b$-axis \\
\hline & & 3.70 & $75 \%$ ER along $a$-axis, $25 \%$ ER along $c$-axis \\
\hline vii & 3.92 & 3.81 & $70 \%$ ER along $a$-axis, $30 \%$ IM (pyranose ring torsion, $\mathrm{CH}_{2} \mathrm{OH}$ group wagging) \\
\hline
\end{tabular}

${ }^{a}$ ET: external translation; ER: external rotation; ED: external deformation; IM: internal motion.

vibrations of molecules. At the same time, it has also been observed that vibration modes such as twisting, swinging, and deformation are present in partial atoms and local groups in monosaccharide molecules. These low-frequency collective vibration modes are complex and closely related to the diversity of carbohydrate molecular conformations. 


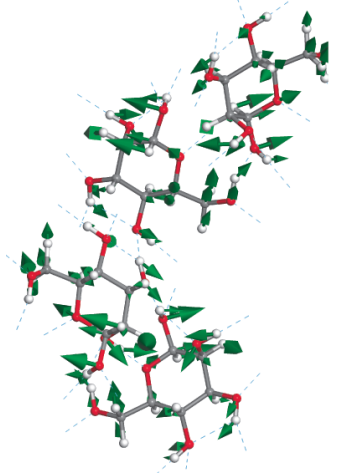

$2.93 \mathrm{THz}$

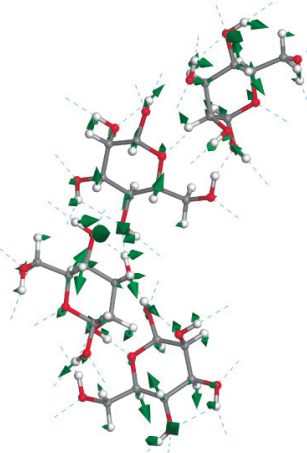

$3.26 \mathrm{THz}$

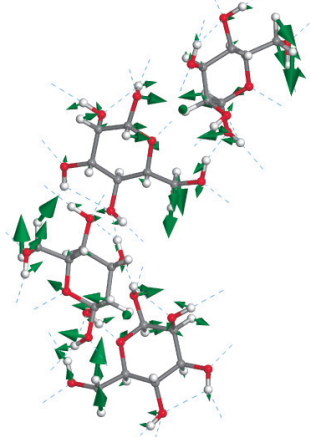

$3.68 \mathrm{THz}$

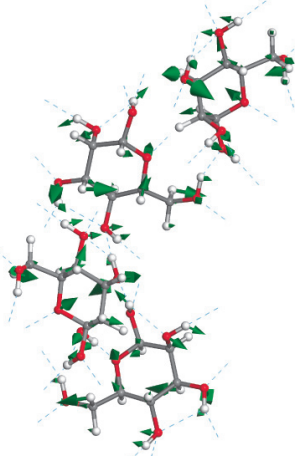

$3.80 \mathrm{THz}$

FIgURE 5: The displacement vector representations of the PBE calculated vibrational modes of mannose at $2.93 \mathrm{THz}, 3.26 \mathrm{THz}, 3.68 \mathrm{THz}$, and $3.80 \mathrm{THz}$.

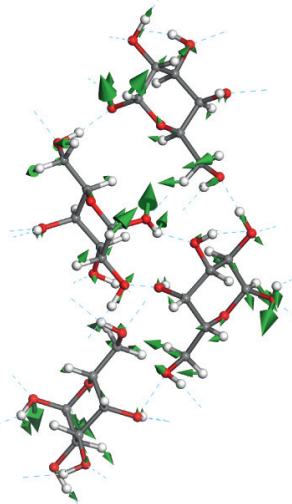

$2.06 \mathrm{THz}$

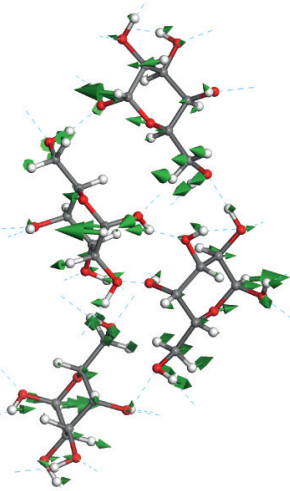

$2.43 \mathrm{THz}$

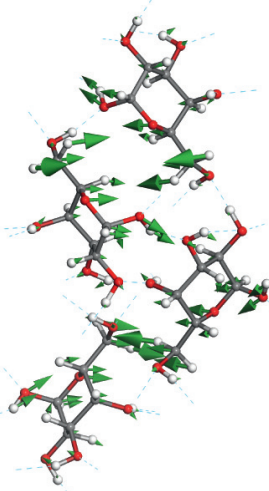

$2.65 \mathrm{THz}$

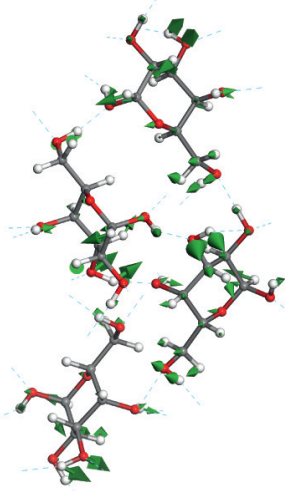

$2.79 \mathrm{THz}$

Figure 6: The displacement vector representations of the PBE calculated vibrational modes of galactose at $2.06 \mathrm{THz}, 2.43 \mathrm{THz}, 2.65 \mathrm{THz}$, and $2.79 \mathrm{THz}$.

\section{Conclusions}

Terahertz spectra of crystalline mannose and galactose were investigated in the spectral range of 0.5 to $4.0 \mathrm{THz}$, and the characteristic absorption peaks were assigned using solidstate DFT lattice dynamics calculations. These two isomers can be easily distinguished by their experimental absorption features, which can be used as fingerprints for detecting and identifying isomers in the terahertz region. It has been demonstrated that the PBE density functional within the GGA level is capable of producing a satisfactory simulation of the observed terahertz spectra of mannose and galactose. The differences in terahertz spectra between the two isomers are mainly due to their different spatial structures and intermolecular interactions. According to the calculation results of PBE, the spectral features observed in the experiments were assigned as primarily the translation, deformation, and rotation of the external lattice and with lesser contributions from intramolecular motions such as the pyranose ring, $\mathrm{CH}_{2} \mathrm{OH}$ group, and hydroxyl group. The results demonstrate that solid-state DFT calculations have the ability to reliably distinguish the subtle differences in the terahertz spectra of similar solid-state systems.

\section{Data Availability}

The data can be obtained from the corresponding author upon request.

\section{Conflicts of Interest}

The authors declare that they have no conflicts of interest.

\section{Acknowledgments}

The authors would like to thank Prof. Peng Wang and Prof. Huiyun Zhang for their valuable comments and assistance in the lab work. The authors also thank P.G. Alice for the help in English writing and proofreading. This work was supported by the National Key Research and Development Program of China (2017YFA0701000).

\section{References}

[1] G. Ren, S. Zong, Z. Zhu et al., "Far-infrared terahertz properties of L-cysteine and its hydrochloride monohydrate," Spectrochimica Acta Part A: Molecular and Biomolecular Spectroscopy, vol. 225, pp. 117476-117481, 2020. 
[2] Y. Liu, T. Zhou, and J.-C. Cao, "Terahertz spectral of enantiomers and racemic amino acids by time-domain-spectroscopy technology," Infrared Physics \& Technology, vol. 96, pp. 17-21, 2019.

[3] T. Gaillard, A. Trivella, R. H. Stote, and P. Hellwig, "Far infrared spectra of solid state L-serine, L-threonine, L-cysteine, and L-methionine in different protonation states," Spectrochimica Acta Part A: Molecular and Biomolecular Spectroscopy, vol. 150, pp. 301-307, 2015.

[4] A. L. Skelbaek-Pedersen, M. Anuschek, T. K. Vilhelmsen, J. Rantanen, and J. A. Zeitler, "Non-destructive quantification of fragmentation within tablets after compression from scattering analysis of terahertz transmission measurements," International Journal of Pharmaceutics, vol. 588, pp. 119769-119777, 2020.

[5] S. Warnecke, J. X. Wu, A. Rinnan et al., "Quantifying crystalline $\alpha$-lactose monohydrate in amorphous lactose using terahertz time domain spectroscopy and near infrared spectroscopy," Vibrational Spectroscopy, vol. 102, pp. 39-46, 2019.

[6] U. Keshwala, S. Rawat, and K. Ray, "Design and analysis of DNA shaped antenna for terahertz and sub-terahertz applications," Optik, vol. 232, pp. 166512-166520, 2021.

[7] F. Qu, Y. Pan, L. Lin et al., "Experimental and theoretical study on terahertz absorption characteristics and spectral de-noising of three plant growth regulators," Journal of Infrared, Millimeter and Terahertz Waves, vol. 39, no. 10, pp. 1015-1027, 2018.

[8] J. Gan, B. Qin, Y. Li, and J. Qiu, "Resolution of overlapping terahertz spectra using non-negative matrix factorization base on pure variables initialization," Optik, vol. 176, pp. 600-610, 2019.

[9] J. Wang, H. Lindley-Hatcher, K. Liu, and E. Pickwell-MacPherson, "Evaluation of transdermal drug delivery using terahertz pulsed imaging," Biomedical Optics Express, vol. 11, no. 8, pp. 4484-4490, 2020.

[10] D. Molter, D. Hübsch, T. Sprenger et al., "Mail inspection based on terahertz time-domain spectroscopy," Applied Sciences, vol. 11, no. 3, pp. 950-961, 2021.

[11] S. Albert, I. Bolotova, Z. Chen et al., "High resolution $\mathrm{GHz}$ and $\mathrm{THz}$ (FTIR) spectroscopy and theory of parity violation and tunneling for 1,2-dithiine (C4H4S2) as a candidate for measuring the parity violating energy difference between enantiomers of chiral molecules," Physical Chemistry Chemical Physics, vol. 18, no. 31, pp. 21976-21993, 2016.

[12] S. J. Dampf and T. M. Korter, "Crystalline molecular standards for low-frequency vibrational spectroscopies," Journal of Infrared, Millimeter and Terahertz Waves, vol. 41, no. 11, pp. 1284-1300, 2020.

[13] Y. Li, L. Xu, Q. Zhou, G. Xiong, Y. Shen, and X. Deng, “A comparative evaluation of the activities of thiol group and hydroxyl group in low-frequency vibrations using terahertz spectroscopy and DFT calculations," Spectrochimica Acta Part A: Molecular and Biomolecular Spectroscopy, vol. 214, pp. 246-251, 2019.

[14] Z. Wu, Z. Zhu, C. Cheng et al., "Terahertz spectroscopy of enantiomeric and racemic pyroglutamic acid," Spectrochimica Acta Part A: Molecular and Biomolecular Spectroscopy, vol. 225, pp. 117509-117513, 2020.

[15] M. R. C. Williams, A. B. True, A. F. Izmaylov, T. A. French, K. Schroeck, and C. A. Schmuttenmaer, "Terahertz spectroscopy of enantiopure and racemic polycrystalline valine," Physical Chemistry Chemical Physics, vol. 13, no. 24, pp. 11719-11730, 2011.
[16] Z.-P. Zheng, W.-H. Fan, H. Li, and J. Tang, "Terahertz spectral investigation of anhydrous and monohydrated glucose using terahertz spectroscopy and solid-state theory," Journal of Molecular Spectroscopy, vol. 296, pp. 9-13, 2014.

[17] T. Zhang, J. Cai, and Z. Zhou, "Terahertz spectroscopic investigation of crystalline $\beta$-D-galactopyranose," Spectroscopy and Spectral Analysis, vol. 28, pp. 721-725, 2008.

[18] C. Du, X. Zhang, and Z. Zhang, "Quantitative analysis of ternary isomer mixtures of saccharide by terahertz time domain spectroscopy combined with chemometrics," $V i$ brational Spectroscopy, vol. 100, pp. 64-70, 2019.

[19] L. Duvillaret, F. Garet, and J.-L. Coutaz, "Highly precise determination of optical constants and sample thickness in terahertz time-domain spectroscopy," Applied Optics, vol. 38, no. 2, pp. 409-415, 1999.

[20] T. D. Dorney, R. G. Baraniuk, and D. M. Mittleman, "Material parameter estimation with terahertz time-domain spectroscopy," Journal of the Optical Society of America A, vol. 18, no. 7, pp. 1562-1571, 2001.

[21] S. J. Clark, M. D. Segall, C. J. Pickard et al., "First principles methods using CASTEP," Zeitschrift für Kristallographie Crystalline Materials, vol. 220, no. 5-6, pp. 567-570, 2005.

[22] E. Patyk, A. Jenczak, and A. Katrusiak, "Giant strain geared to transformable H-bonded network in compressed $\beta$-d-mannose," Physical Chemistry Chemical Physics, vol. 18, no. 16, pp. 11474-11479, 2016.

[23] B. Sheldrick, "The crystal structures of the $\alpha$ - and $\beta$-anomers of D-galactose," Acta Crystallographica Section B Structural Crystallography and Crystal Chemistry, vol. 32, no. 4, pp. 1016-1020, 1976.

[24] J. P. Perdew, K. Burke, and M. Ernzerhof, "Generalized gradient approximation made simple," Physical Review Letters, vol. 77, no. 18, pp. 3865-3868, 1996.

[25] H. J. Monkhorst and J. D. Pack, "Special points for Brillouinzone integrations," Physical Review B, vol. 13, no. 12, pp. 5188-5192, 1976.

[26] J. P. Perdew, J. A. Chevary, S. H. Vosko et al., “Atoms, molecules, solids, and surfaces: applications of the generalized gradient approximation for exchange and correlation," Physical Review B, vol. 46, no. 11, pp. 6671-6687, 1992.

[27] T. Lu and F. Chen, "Multiwfn: a multifunctional wavefunction analyzer," Journal of Computational Chemistry, vol. 33, no. 5, pp. 580-592, 2012.

[28] P. Jacqueline and G. Pandraud, "Structure Cristalline de l' $\alpha$ D-Galactose," Acta Crystallographica Section B, vol. 32, pp. 2810-2813, 1976. 